\title{
The Present and the Future of JAERI Recoil Mass Separator
}

\author{
S. Mitsuoka, ${ }^{*}$ H. Ikezoe, K. Nishio, K. Satou, and L. Dan \\ Japan Atomic Energy Research Institute, Tokai, Ibaraki 319-1195, Japan
}

Received: February 1, 2002; In Final Form: April 5, 2002

A recoil mass separator has been in operation at the JAERI tandem-booster facility since 1995, for in-flight separation of low-yield reaction products recoiling out in the beam direction. The current status and future plan together with a brief description of the design performance are presented.

\section{Introduction}

The JAERI Recoil Mass Separator (JAERI-RMS) was constructed at the tandem-booster facility at the end of March in 1995. Immediately after the performance test, ${ }^{1,2}$ the RMS was utilized for various experiments where extremely low-yield reaction products of sub-nb cross sections were separated in flight from the high flux of the primary beam and other reaction products. At an early stage of the experiments, the RMS was used to separate new neutron deficient isotopes in order to study the decay properties of short-lived $\alpha$-emitting isotopes near the proton drip line in the light actinide region. ${ }^{3,4}$ For the next three years, the excitation functions of evaporation residue cross sections were measured in order to study the reaction mechanism of sub-barrier fusion of well-deformed nuclei. ${ }^{5.6}$ In such experiments, it is important to collect rare fusion products with high transport efficiency in wide charge distribution while keeping low background. For this purpose, the RMS will be improved in this year by installing a new quadrupole magnet. And recently, for another type of research in nuclear astrophysics, a test experiment has been started to separate low-energy radioactive nuclear beam (RNB) by using the RMS. ' In future, more intense RNB will be supplied to the RMS beam line from a newly planned upgrade tandem-booster facility. ${ }^{8}$

In the next section, the ion optical design and performance of the RMS is described. Short descriptions of the experiments which have been or are being carried out by using the RMS is presented in sect. 3. The future plan is briefly introduced in sect. 4.

\section{Ion Optical Design and Performance}

The schematic layout of the RMS is shown in Figure 1 and the ion optical properties are listed in Table 1. The RMS has a symmetric configuration of $\mathrm{Q}_{1} \mathrm{Q}_{2}-\mathrm{ED}_{1}-\mathrm{MD}-\mathrm{ED}_{2}-\mathrm{Q}_{3} \mathrm{Q}_{4}-\mathrm{O}$. Two $25^{\circ}$ electric dipoles $\left(\mathrm{ED}_{1}\right.$ and $\left.\mathrm{ED}_{2}\right)$ and one $-50^{\circ}$ magnetic dipole (MD) disperse reaction products by their mass/charge $(m / q)$ ratio and cancel out the energy/charge dispersion. The design value of $m / q$ dispersion is $0 \sim 1.5 \mathrm{~cm} / \%$ (variable). Two quadrupole doublets $\left(\mathrm{Q}_{1} \mathrm{Q}_{2}\right.$ and. $\left.\mathrm{Q}_{3} \mathrm{Q}_{4}\right)$ allow to focus the recoils irrespective of their angular spread and also to change the $m / q$ dispersion at the focal planes. The octupole magnet $(O)$ is for the correction of a non-linearity of the $m / q$ dispersion. These electric and magnetic components are arranged on a rotating platform $\left(-5^{\circ}\right.$ to $\left.40^{\circ}\right), 9.4 \mathrm{~m}$ in length.

The RMS was designed especially to reduce a background originating from the scattered beams from the anode surface of the $\mathrm{ED}_{1}{ }^{1}{ }^{1}$ For this purpose, the $\mathrm{ED}_{1}$ anode is vertically split into two parts separated by $1 \mathrm{~cm}$, so that the primary beam can pass through this gap without hitting the anode surface. The slit anode also allow to monitor a beam current during the experiment by a Faraday cup located behind the $\mathrm{ED}_{1}$ anode.

The performance of the RMS was tested, ${ }^{1}$ by using $\alpha$ particles

*Corresponding author. E-mail: mitsuoka@nucleus3.tokai.jaeri.go jp. FAX: +81-29-282-5927. from an ${ }^{241} \mathrm{Am}$ source and beams of ${ }^{127} \mathrm{I}$ and ${ }^{28} \mathrm{Si}$ from the tandem accelerator. $A^{\text {nat }} \mathrm{Ag}$ target was bombarded with ${ }^{127} \mathrm{I}$ beam of $120 \mathrm{MeV}$ and the recoiling $\mathrm{Ag}$ particles at $\theta=20^{\circ}$ were led to the RMS. The particles passing through the RMS were detected by a position sensitive silicon detector $(45 \mathrm{~mm} \times 8 \mathrm{~mm}$ ) at the focal plane. It was confirmed that the mass 107 and 109 of $\mathrm{Ag}$ isotopes with the same charge state $25^{+}$were separated with no background. Fusion evaporation residues were also separated at $\theta=0^{\circ}$ (the beam direction) in the reactions of ${ }^{28} \mathrm{Si}+{ }^{59} \mathrm{Co},{ }^{89} \mathrm{Y},{ }^{\text {nat }} \mathrm{Ag}$, ${ }^{\text {nat }}$ In at 130 and $150 \mathrm{MeV}$. The mass resolution was obtained to be $A / \Delta A \simeq 300$, which is nearly equal to the calculated value. The capabilities of the background suppression were $3 \times 10^{10}$ for ${ }^{59} \mathrm{Co}, 4.5 \times 10^{10}$ for ${ }^{89} \mathrm{Y}, 3 \times 10^{11}$ for ${ }^{\text {nat }} \mathrm{Ag}$, and $2 \times 10^{12}$ for ${ }^{\text {nat }}$ In targets. This means that the split anode adopted in the RMS is really effective on the reduction of beam background.

The solid angle and transport efficiency of the RMS were measured, ${ }^{2}$ by using elastic recoils of ${ }^{197} \mathrm{Au}$ in ${ }^{28} \mathrm{Si}+{ }^{197} \mathrm{Au}$ and also $5.486 \mathrm{MeV} \alpha$ from a source. The obtained values of solid angle were 11 and $21 \mathrm{msr}$ for the focusing conditions with mass dispersion of 1.1 and $0 \mathrm{~cm} / \%$, respectively. The energy acceptance was measured by changing the energy setting of the RMS in small steps after selecting a charge state, and the mass acceptance was measured by changing the charge state with a constant energy. Good agreements between the data and the ion-optical calculation were obtained.

The absolute values of transport efficiency were measured ${ }^{2}$ in ${ }^{30} \mathrm{Si}+{ }^{141} \mathrm{Pr}$ reaction by detecting $\gamma$ rays of long-lived evaporation residues of ${ }^{167} \mathrm{Lu}$ and ${ }^{167} \mathrm{Yb}$ with the charge state $q=18^{+}$, which were implanted into a catcher foil placed just behind the target or at the focal plane of the RMS. The charge-state distributions were also measured and compared with available phenomenological formulas. The absolute transport efficiency multiplied by the charge fraction was consistent with the calculated result.

\section{Experiments}

At an early stage of experiments using the RMS, new neutron deficient isotopes ${ }^{209} \mathrm{Th}$ (Ref. 3) and ${ }^{212} \mathrm{~Pa}$ (Ref. 4) were produced via hot fusion reactions of ${ }^{182} \mathrm{~W}\left({ }^{32} \mathrm{~S}, 5 n\right)$ at $171 \mathrm{MeV}$ and ${ }^{182} \mathrm{~W}\left({ }^{35} \mathrm{Cl}, 5 n\right)$ at $183 \mathrm{MeV}$, respectively. The fusion evaporation residues separated by the RMS were implanted into a doublesided position sensitive silicon detector $(73 \mathrm{~mm} \times 55 \mathrm{~mm})$. The energies and two-dimensional positions of the implanted evaporation residues and those of the $\alpha$ particles in their subsequent $\alpha$-decay chains were registered together with the times associated with the detection. The observed short-lived activities were identified on the basis of time- and position-correlated $\alpha$-decay chains. The $\alpha$-decay energies and half-lives were determined to be $8.080(50) \mathrm{MeV}$ and $3.8_{-1.5}^{+6.9} \mathrm{~ms}$ for ${ }^{209} \mathrm{Th}$, and $8.270(30) \mathrm{MeV}$ and $5.1_{-1.9}^{+6.1} \mathrm{~ms}$ for ${ }^{212} \mathrm{~Pa}$. The production cross sections were $1 \mathrm{nb}$ for ${ }^{209} \mathrm{Th}$ and $0.5 \mathrm{nb}$ for ${ }^{212} \mathrm{~Pa}$, when the RMS transport efficiency of $19 \%$ was assumed. In the same manner, we have observed (i) a new spontaneous fission decay of ${ }^{264} \mathrm{Sg}$ produced in ${ }^{238} \mathrm{U}\left({ }^{30} \mathrm{Si}, 4 n\right)$ at $158 \mathrm{MeV},{ }^{9}$ (ii) a new isomeric state in ${ }^{217} \mathrm{~Pa}$ 


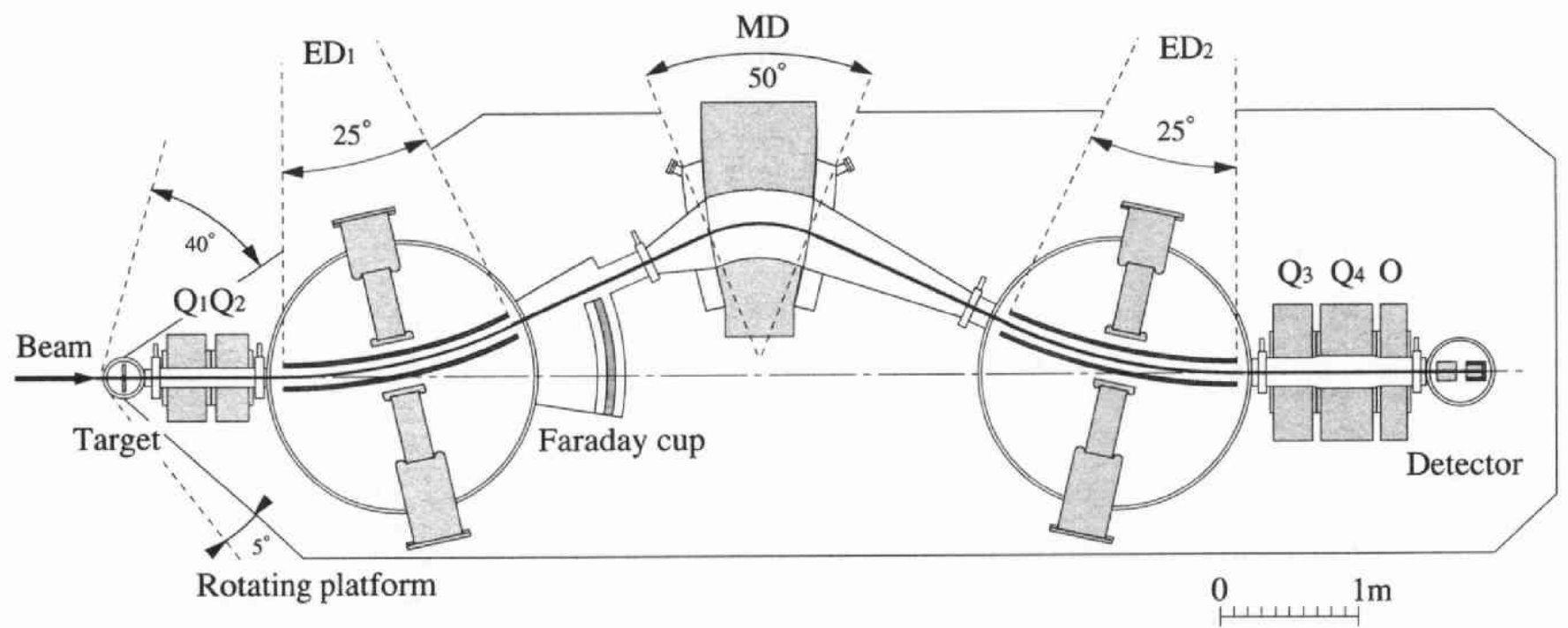

Figure 1. Schematic drawing of the JAERI-RMS.

TABLE 1: The dimensions and ion-optical properties of the JAERI-RMS.

\begin{tabular}{|c|c|c|c|c|c|}
\hline Energy acceptance & \multicolumn{2}{|c|}{ $\pm 12 \%$} & & & \\
\hline Mass acceptance $(m / q)$ & \multicolumn{2}{|c|}{ $\pm 4 \%$} & & & \\
\hline Mass resolution & \multicolumn{2}{|l|}{300} & & & \\
\hline Mass dispersion & \multicolumn{2}{|c|}{$1.5 \mathrm{~cm} / \%$} & \multicolumn{3}{|c|}{ (variable) } \\
\hline Solid angle & \multicolumn{2}{|c|}{$21 \mathrm{msr}$} & \multicolumn{3}{|c|}{ (variable) } \\
\hline $\mathbf{M}_{x}$ magnification & \multicolumn{2}{|c|}{1.66} & \multicolumn{3}{|c|}{ (variable) } \\
\hline $\mathbf{M}_{\mathbf{y}}$ magnification & \multicolumn{2}{|c|}{1.17} & \multicolumn{3}{|c|}{ (variable) } \\
\hline Platform rotation angle & \multicolumn{2}{|c|}{$-5^{\circ} \sim 40^{\circ}$} & & & \\
\hline Overall length & \multicolumn{2}{|c|}{$9.4 \mathrm{~m}$} & & & \\
\hline Dipoles & \multicolumn{2}{|c|}{ MD } & \multicolumn{3}{|c|}{$E D_{1}$ and $E D_{2}$} \\
\hline Radius of curvature & \multicolumn{2}{|l|}{$1 \mathrm{~m}$} & \multicolumn{3}{|l|}{$4 \mathrm{~m}$} \\
\hline Deflection angle & \multicolumn{2}{|l|}{$50^{\circ}$} & \\
\hline Radius of EFB* & \multicolumn{2}{|c|}{$3.9 \mathrm{~m}$} & \multicolumn{3}{|l|}{$25^{\circ}$} \\
\hline EFB $^{*}$ shim angle & \multicolumn{2}{|c|}{$16^{\circ}$} & \multicolumn{3}{|l|}{$0^{\circ}$} \\
\hline Gap & \multicolumn{2}{|c|}{$12 \mathrm{~cm}$} & \multicolumn{3}{|c|}{$12 \mathrm{~cm}$} \\
\hline Maximum rigidity & \multicolumn{2}{|c|}{$10 \mathrm{kGm}$} & \multicolumn{3}{|c|}{$20 \mathrm{MV}$} \\
\hline Quadrupoles and Octupole & $\mathrm{Q}_{1}$ & $\mathrm{Q}_{2}$ & $\mathrm{Q}_{3}$ & $\mathrm{Q}_{4}$ & $\mathrm{O}$ \\
\hline Bore diameter $/ \mathrm{cm}$ & 10 & 10 & 15 & 18 & 15 \\
\hline Effective length $/ \mathrm{cm}$ & 30 & 20 & 30 & 40 & 15 \\
\hline Maximum field gradient & 1.3 & 1.3 & 0.8 & 0.8 & 7.1 \\
\hline$/ \mathrm{kG} \cdot \mathrm{cm}^{-1}$ for $\mathrm{Q}, \mathrm{kG} \cdot \mathrm{cm}$ & for $\mathrm{O}$ & & & & \\
\hline
\end{tabular}

produced in ${ }^{194} \mathrm{Pt}\left({ }^{28} \mathrm{Si}, 5 n\right)$ at $163 \mathrm{MeV},{ }^{10}$ and (iii) $\alpha$ decay of ${ }^{217} \mathrm{Th}$ populating the low-lying two excited states in ${ }^{213} \mathrm{Ra}$ in ${ }^{28} \mathrm{Si}+{ }^{198} \mathrm{Pt}$ reaction. ${ }^{11}$

For the last three years, we have investigated the sub-barrier fusion between heavy projectiles and well-deformed targets in the reactions of ${ }^{60} \mathrm{Ni}+{ }^{154} \mathrm{Sm}$ and ${ }^{32} \mathrm{~S}+{ }^{182} \mathrm{~W}$ (Ref. 5), and ${ }^{76} \mathrm{Ge}+{ }^{150} \mathrm{Nd}$ and ${ }^{28} \mathrm{Si}+{ }^{198} \mathrm{Pt}$ (Ref. 6), where the same compound nucleus ${ }^{214} \mathrm{Th}$ and ${ }^{226} \mathrm{U}$ are formed, respectively. This is aimed to study an appealing speculation of gentle fusion ${ }^{12}$ or hugging fusion, ${ }^{13}$ which were theoretically proposed as a novel way to synthesize the superheavy elements. It was found that the fusion probability depends strongly on the orientation of nuclear deformation. The measured excitation functions showed the large fusion hindrance in the low energy region where only the collision at the tip of the deformed target is possible, while no fusion hindrance was observed at higher energies where the side collisions become possible. This supports the original idea of hugging fusion that the compact configuration at side collision is more favorable for the complete fusion than the elongated configuration at tip collision. Moreover, we have studied (i) the effect of nuclear deformation in ${ }^{76} \mathrm{Ge}+{ }^{150} \mathrm{Nd}$ reaction by directly comparing with the case of spherical projectile and target combina- tion in ${ }^{82} \mathrm{Se}+{ }^{\text {nat }} \mathrm{Ce}$ reaction, ${ }^{14}$ (ii) the effect of neutron number in ${ }^{60,64} \mathrm{Ni}+{ }^{154} \mathrm{Sm}$ reactions to see how the $n$-rich projectile acts on the fusion probability, ${ }^{15}$ and (iii) the effect of nuclear shell structure in ${ }^{82} \mathrm{Se}+{ }^{134,138} \mathrm{Ba}$ reactions. ${ }^{16}$ The details of these experimental results are shown by Ikezoe et al. elsewhere in this proceedings.

Recently, as another type of experiments by using the RMS, we started direct-exclusive measurements in astrophysical reactions like $(\alpha, n)$ or $(\alpha, p)$ with radioactive nuclei of ${ }^{8} \mathrm{Li},{ }^{10} \mathrm{Be}$, ${ }^{12} \mathrm{~B},{ }^{14,15} \mathrm{C}$, or ${ }^{16,17} \mathrm{~N}$ (Ref. 7). Especially, ${ }^{8} \mathrm{Li}(\alpha, n)$ is the key reaction for nucleosynthesis reaction network of r-process in Supernovae. ${ }^{7}$ Radioactive nuclear beams (RNBs) are produced via nuclear transfer reaction, for example, $(d, p)$ reaction by irradiating deuteron gas target with heavy-ion beams from Tandem accelerator, and then separated from the primary beam by the RMS. Low-energy RNBs are implanted into a multiplesampling and tracking proportional chamber (MSTPC), ${ }^{17}$ which is filled with helium gas working as both the detector gas and the target for the secondary reaction. The neutron TOF is measured by a neutron detector array surrounding the MSTPC. The analysis of the first experiment in $\alpha\left({ }^{16} \mathrm{~N},{ }^{19} \mathrm{~F}\right) n$ is in progress.

\section{The Future Plan}

We have two plans (i) to improve the transport efficiency of the RMS for wide charge distribution and (ii) to supply more intense RNBs as well as intense stable nuclear beams to the RMS beam line.

As for the plan (i), a new quadrupole singlet will be installed, in this year, between the magnetic dipole (MD) and the second electric dipole $\left(\mathrm{ED}_{2}\right)$. In general use of the RMS, fusion products of interest with another charge states can not pass the gap of $\mathrm{ED}_{2}$, due to the mass/charge $(m / q)$ dispersion produced by MD. The new $Q$ magnet bends horizontally the $m / q$ dispersed particles to the central axis. It is estimated that evaporation residue of $\mathrm{Sg}(Z=106)$ produced in $\mathrm{Kr}+\mathrm{Yb}$ reaction, for example, with five charge states can be transported to the focal plane of the RMS,

As for the plan (ii), JAERI and KEK (High Energy Accelerator Research Organization) has jointly proposed a new RNB project to produce $\mathrm{RNBs}(0.1 \sim 8 \mathrm{MeV} / \mathrm{u})$ with high quality and sufficient intensity for studying nuclear physics, nuclear astrophysics, material science, and so on. ${ }^{8}$ This joint project has been started since this year FY2001 to construct an ISOL-based RNB facility at JAERI by utilizing the existing accelerators of both the JAERI tandem-booster and the KEK-Tanashi RNB linac complex. The RNB with energy up to $1 \mathrm{MeV} / \mathrm{u}$ will be available from FY2004. After the next two years, a pre-booster linac will 
be installed to increase the RNB energy up to $8 \mathrm{MeV} / \mathrm{u}$ by the existing superconducting booster linac. Intense stable nuclear beams will be also available from the linac complex independent of the tandem for studying, for example, SHE synthesis by using the RMS.

Acknowledgement. The authors would like to acknowledge the accelerator crew of JAERI tandem facility.

\section{References}

(1) H. Ikezoe, Y. Nagame, T. Ikuta, S. Hamada, I. Nishinaka, and T. Ohtsuki, Nucl. Instrum. Methods A 376, 420 (1996); H. Ikezoe, T. Ikuta, S. Mitsuoka, S. Hamada, Y. Nagame, I. Nishinaka, Y. Tsukada, Y. Oura, and T. Ohtsuki, Nucl. Instrum. Methods B 126, 340 (1997).

(2) T. Kuzumaki, H. Ikezoe, S. Mitsuoka, T. Ikuta, S. Hamada, Y. Nagame, I. Nishinaka, T. Ohtsuki, and O. Hashimoto, Nucl. Instrum. Methods A 437, 107 (1999).

(3) H. Ikezoe, T. Ikuta, S. Hamada, Y. Nagame, I. Nishinaka, K. Tsukada, and Y. Oura, Phys. Rev. C 54, 2043 (1996).

(4) S. Mitsuoka, H. Ikezoe, T. Ikuta, Y. Nagame, K. Tsukada, I. Nishinaka, Y. Oura, and Y. L. Zhao, Phys. Rev. C 55, 1555 (1997).

(5) S. Mitsuoka, H. Ikezoe, K. Nishio, and J. Lu, Phys. Rev. C 62, 054603 (2000).

(6) K. Nishio, H. Ikezoe, S. Mitsuoka, and J. Lu, Phys. Rev. C 62, 014602 (2000).

(7) H. Ishiyama, JAERI-Conf 2001-014, 13 (2001); Y. Mizoi, T. Fukuda, Y. Matsuyama, T. Miyachi, H. Miyatake, N. Aoi, N. Fukuda, M. Notani, Y. X. Watanabe, K. Yoneda, M. Ishihara, H. Sakurai, Y. Watanabe, and A. Yoshida, Phys. Rev. C 62, 065801 (2000).
(8) H. Miyatake, S. Arai, Y. Arakaki, Y. Fuchi, H. Ishiyama, S. C. Jeong, I. Katayama, H. Kawakami, K. Niki, T. Nomura, M. Okada, M. Oyaizu, Y. Takeda, M. H. Tanaka, E. Tojyo, M. Tomizawa, N. Yoshikawa, S. Abe, S. Hanashima, K. Horie, S. Ichikawa, H. Iimura, H. Ikezoe, T. Ishii, N. Ishizaki, A. Iwamoto, A. Iwase, S. Kanda, M. Koizumi, M. Matsuda, S. Mitsuoka, Y. Nagame, K. Nishio, I. Ohuchi, A. Osa, M. Oshima, M. Sataka, S. Takeuchi, H. Tayama, K. Tsukada, Y. Tsukihashi, and T. Yoshida, Proceedings of the Japan-Italy Joint Symposium on Heavy Ion Physics, Sep. 26-29, 2001 (World Scientific, Singapore, to be published).

(9) H. Ikezoe, T. Ikuta, S. Mitsuoka, Y. Nagame, I. Nishinaka, K. Tsukada, T. Ohtsuki, T. Kuzumaki, and J. Lu, Eur. Phys. J. A 2, 379 (1998).

(10) T. Ikuta, H. Ikezoe, S. Mitsuoka, I. Nishinaka, K. Tsukada, Y. Nagame, and J. Lu, Phys. Rev. C 57, R2804 (1998).

(11) K. Nishio, H. Ikezoe, S. Mitsuoka, and J. Lu, Phys. Rev. C 61, 034309 (2000).

(12) W. Nörenberg, Proceedings of the International Workshop on Heavy-Ion Fusion, Padova, Italy, 1994.

(13) A. Iwamoto, P. Möller, J. R. Nix, and H. Sagawa, Nucl. Phys. A 596, 329 (1996).

(14) K. Nishio, H. Ikezoe, S. Mitsuoka, K. Satou, and S. C. Jeong, Phys. Rev. C 63, 044610 (2000).

(15) S. Mitsuoka, H. Ikezoe, K. Nishio, K. Satou, and J. Lu, Phys. Rev. C 65, 054608 (2002).

(16) K. Satou, H. Ikezoe, S. Mitsuoka, K. Nishio, and S. C. Jeong, Phys. Rev. C 65, 054602 (2002).

(17) Y. Mizoi, T. Fukuda, Y. Matsuyama, T. Miyachi, J. Nakano, N. Fukuda, M. Hirai, H. Kobayashi, Y. X. Watanabe, H. Sakurai, Y. Watanabe, and A. Yoshida, Nucl. Instrum. Methods A 431, 112 (1999). 